\title{
Effects of Gender, Age, and Clot Formation on the Measurements of Tryptophan Metabolites in Blood
}

\author{
Junichi Masuda1, Masato Karayama², Takafumi Suda², Masato Maekawa ${ }^{3}$, Fumiko Shimizu, \\ Akikazu Takada ${ }^{*}$ \\ ${ }^{1}$ Global Application Development Center, Shimadzu Corporation, Kanagawa-ken, Japan \\ ${ }^{2}$ Second Department of Internal Medicine, Hamamatsu University School of Medicine, Hamamatsu, Japan \\ ${ }^{3}$ Department of Laboratory Medicine, Hamamatsu University School of Medicine, Hamamatsu, Japan \\ ${ }^{4}$ Faculty of Life and Environmental Sciences, Showa Women's University, Tokyo, Japan \\ ${ }^{5} 1$ nternational Projects on Food and Health (NPO), Tokyo, Japan \\ Email: *takadaa@mwd.biglobe.ne.jp
}

How to cite this paper: Masuda, J., Karayama, M., Suda, T., Maekawa, M., Shimizu, F. and Takada, A. (2019) Effects of Gender, Age, and Clot Formation on the Measurements of Tryptophan Metabolites in Blood. Food and Nutrition Sciences, 10, 761-775.

https://doi.org/10.4236/fns.2019.107056

Received: June 11, 2019

Accepted: July 26, 2019

Published: July 29, 2019

Copyright (c) 2019 by author(s) and Scientific Research Publishing Inc. This work is licensed under the Creative Commons Attribution International License (CC BY 4.0).

http://creativecommons.org/licenses/by/4.0/

\begin{abstract}
Background: Tryptophan metabolism plays important roles in health and diseases. Although simultaneous measurements of tryptophan metabolites are successfully measured, influences of age, gender, and clot formation on the measurements have not been reported. Methods: We took blood from young and old Japanese men and women and compared plasma levels of tryptophan metabolites. We also took plasma and serum from the blood of middle-aged men $(\mathrm{n}=10)$. Metabolites analysis was performed by a liquid chromatograph tandem mass spectrometer, the LCMS-8060 quadrupole mass spectrometer combined with Nexera X2 liquid chromatograph system (Shimadzu Corporation, Kyoto, Japan). Body mass index (BMI) and TRP metabolites have been measured in healthy young men $(n=48)$, young women $(n=47)$, old men ( $n$ $=44)$, and old women $(\mathrm{n}=39)$. TRP metabolites were measured by using the ultrahigh speed liquid chromatography-mass spectroscopy (Shimadzu Corporation). Results: Tryptophan and its metabolites such as serotonin, 5-hydroxyindole acetic acid, indole-3-acetic acid, kynurenine, anthranilic acid, were higher in young women and old men than young men and old women. Plasma levels of 3-hydroxykynurenine and xanthurenic acid were lower in young women and old men. Comparison of plasma and serum indicates that most of metabolites were higher in serum than plasma except for 3-hydroxy-kynurenine and quinolinic acid. Conclusion: Metabolites of the upper stream of degradation of tryptophan were higher in young women and old men than young men and old women, which suggests that the degradation of tryptophan was accelerated in young men and old women than young
\end{abstract}


women and old men. Serum preparation may activate tryptophan degradation resulting in higher levels of metabolites in serum than in plasma.

\section{Keywords}

Plasma, Serum, Platelet, Tryptophan, Serotonin, 5-Hydroxyindole Acetic

Acid, Kynurenine, 3-Hydroxykynurenine, Kynurenic Acid, Anthranilic Acid, Xanthurenic Acid, Indole-3-Acetic Acid

\section{Introduction}

Tryptophan (TRP) is an essential amino acid important for protein synthesis, but it also becomes a substrate for the generation of several important bioactive compounds. Probably the best-known fate of TRP is its conversion to serotonin (5-HT; 5-hydroxytryptamine), an important neurotransmitter considered to be involved in the control of many responses in the central nervous system (CNS) and linked to alterations in mood, anxiety, or cognition [1]. Many drugs for depression have been proposed to increase serotonin in neurotransmission. Serotonin can be further converted to N-acetylserotonin (NAS) and melatonin, influencing circadian rhythmicity, one of the biological roles for TRP metabolites [2]. However, in mammals, the majority of free TRP are degraded through the kynurenine (KYN) pathway and generate many metabolites involved in inflammation, immune response, and excitatory neurotransmission [3]. We have shown that foot shock applied to rats resulted in an increase of not only brain 5-HT levels but KYN levels in plasma, kidney, liver and every part of the brain [4] [5] [6]. Several TRP metabolites have been shown to exhibit neuroexcitatory, convulsant, and toxic properties [7] [8]. In the periphery, only $1 \%$ of dietary tryptophan was converted to serotonin and more than $95 \%$ was metabolized to kynurenines [9] [10].

We have recently shown that plasma levels of $5-\mathrm{HT}$ were very low in patients of depression, but 5-HIAA and kynurenine levels were not changed, indicating that the activation of tryptophan in the 5-HT and KYN pathways was not enhanced in depression, but the conversion of 5-HT to 5-HIAA was enhanced [11]. We also showed that plasma levels of TRP metabolites were enhanced in patients of type2 diabetes mellitus (DM) [12] [13] [14].

Since the technology to measure TRP metabolites simultaneously is so important, we thought that we should examine age and gender differences in their plasma levels and effects of clot formation, thus platelet activation on the measurements.

\section{Methods}

1) Samples

We asked male and female acquaintances older than 50 years old and male 
and female college students to participate in the experiments. Acquaintances mean that these participants are personal friends of our group member. The sample sizes and ages of participants are as follows. Old men $(n=25$, age; $60.8 \pm$ 9.9) and old women $(n=39$, age; $67.4 \pm 7.5)$ and young men $(n=49$, age; $20.7 \pm$ 1.5 ) and young women ( $\mathrm{n}=47$, age; $21.2 \pm 0.7)$ Young men are students of Tokyo Institute of Technology and young women are students of Showa Women's college. We obtained informed consent prior to conducting the protocol which had been approved by the Ethical Committee of Showa Women's University and Saiseikai main Hospital. Drs. K. Matsuoka and K. Kato, who are internists checked their health carefully and examined their blood samples and recruited them if there were no health problems such as diabetes, hypertension or not serious diseases experienced in the past. They did not smoke in the past. Exclusion criteria are as follows. We excluded people who took drugs for dyslipidemia, hyperglycemia, or hypertension. Their disease histories were average of 5 years. They were mainly treated by the controls of lifestyles such as foods intakes and exercises. Nobody used insulin for treatments. Some took metabolism promoting drugs such as metformin. Doctors went to colleges to check the health of young participants. We collected blood samples early morning. Participants were asked not to eat anything after 21.00 PM the previous evening. Plasma specimens were collected for assays of blood parameters. We obtained informed consent prior to conducting the protocol which had been approved by the Ethical Committee of Showa Women's University and Saiseikai Shibuya Satellite Clinic. Plasma factors were measured after plasma was separated from the blood. Ethylenediaminetetraacetic acid (EDTA) was used as an anticoagulant.

Table 1 shows the backgrounds of the participants.

2) Measurements of Various Plasma Factors

Blood glucose levels were measured by a hexokinase UV method. Insulin was measured by the CLEIA (chemiluminescent immunoassay) method. We also measured glycemic indexes after giving glucose and sucrose to participants, so that we did not use HbA1c as a marker of glycemia. Lipid and lipoprotein concentrations such as total cholesterol, HDL (high-density lipoprotein cholesterol), LDL (low-density lipoprotein) cholesterol, TG (triglyceride) were determined using a Polychem Chemistry Analyzer (Polymedco Inc.) FFA (free fatty acid) and the concentrations of $\omega$ fatty acids such as AA (arachidonic acid), DHA (docosahexaenoic acid), and EPA (eicosapentaenoic acid) were measured by a

Table 1. The background of healthy young and old men and women.

\begin{tabular}{ccccc}
\hline Subjects & $\begin{array}{c}\text { young men } \\
\mathrm{n}=48\end{array}$ & $\begin{array}{c}\text { young women } \\
\mathrm{n}=47\end{array}$ & $\begin{array}{c}\text { Old men } \\
\mathrm{n}=44\end{array}$ & $\begin{array}{c}\text { Old women } \\
\mathrm{n}=39\end{array}$ \\
\hline Age (years) & $20.7 \pm 1.5$ & $21.2 \pm 0.7$ & $62.4 \pm 9.6$ & $67.4 \pm 7.5$ \\
Height $(\mathrm{m})$ & $1.72 \pm 0.06$ & $1.58 \pm 0.06$ & $1.68 \pm 0.07$ & $1.57 \pm 0.06$ \\
Weight $(\mathrm{kg})$ & $65.1 \pm 9.2$ & $50.9 \pm 5.8$ & $68.8 \pm 10.9$ & $50.6 \pm 6.8$ \\
BMI & $22.1 \pm 3.2$ & $20.3 \pm 1.6$ & $24.3 \pm 3.2$ & $20.5 \pm 2.5$ \\
\hline
\end{tabular}


gas chromatography.

Remnant lipoproteins (RLPs) were isolated from the serum to an immunoaffinity mixed gel containing anti-apolipoprotein A1 and antiapolipoprotein B100 monoclonal antibodies (Japan Immunoresearch Laboratories, Takasaki, Japan), and the cholesterol and triglyceride concentrations of the unbound fraction were measured as RLP cholesterol and RLP-triglyceride, respectively.

3) Measurements of TRP Metabolites

The standard materials of fifteen major tryptophan metabolites such as TRP, L-5-Hydroxytryptophan, 5-HT, L-Kynurenine (KYN), 3-Hyroxykinurenine (3-HKYN), 5-Hydroxytryptophol (5-HTOL), tryptophol (TOL), Kynurenic acid (KA), Quinaldic acid (QA), Xanthurenic acid (XA), 5-Hydroxyindole-acetic acid (HIAA), Indole 3-acetic acid (IAA), 3-Hydroxyanthranilic acid (HAA), Anthranilic acid (AA), Indole-3-lactic acid (ILA) are special grade or biochemical grade regents purchased from Wako Pure Chemical Industries, Ltd. (Osaka, Japan). Formic acid and acetonitrile are LC/MS grade regents and other major chemicals such as methanol are special grade purchased from Wako Chemical as well. Water was obtained as occasion demands from the installed Milli-Q water purification system (Merck-Millipore, MA, USA) Each standard mother solution was prepared as $1000 \mathrm{mg} / \mathrm{L}$ from each regent above in water and/or $0.1 \mathrm{~mol} / \mathrm{L}$ sodium hydroxide aqueous solution. Standard mixture solution for each concentration level of a calibration curve was prepared by dilution of the mother solution with water. Tryptophan metabolites were analyzed by Shimadzu LCMS-8060 or 8050, which consists of Nexera UHPLC systems such as solvent delivery units, an autosampler and column oven, and liquid chromatograph mass spectrometer LCMS-8060 or 8050 with its electro spray interface. The HPLC column used was L-column 2 ODS $(2.1 \mathrm{~mm} \times 150 \mathrm{~mm}, 3 \mu \mathrm{m}$, CERI; Chemical Evaluation and Research Institute, Tokyo, Japan) with a gradient elution of $0.1 \%$ formic acid/acetonitrile system. Before analyses, the condition of multi-reaction monitoring (MRM) was optimized using each standard solution, respectively. Gradient elution was performed by the high-pressure binary gradient program, $5 \%$ of 0.1 formic acid aqueous solution fold in 3 min with acetonitrile, $5 \%$ to $95 \%$ of $0.1 \%$ formic acid aqueous solution in the next 6 min followed by $95 \%$ hold in another $3 \mathrm{~min}$ at flow rate $0.4 \mathrm{~mL} / \mathrm{min}$ under $40^{\circ} \mathrm{C}$. Ionization was executed by an electrospray (ESI) positive mode at $150^{\circ} \mathrm{C}$ (the desolvation line) and $400^{\circ} \mathrm{C}$ (the interface) with $3 \mathrm{~L} / \mathrm{min}$ nebulizing and $5 \mathrm{~L} / \mathrm{min}$ drying gas. Chromatographic data were obtained by MRM mode under optimized transition. Sample pretreatment The samples were stored at $-80^{\circ} \mathrm{C}$. $50 \mu \mathrm{L}$ aliquot of the blood plasma sample was took in $1.5 \mathrm{ml}$ test tube, and then $25 \mu \mathrm{L} 0.1 \%$ formic acid aqueous solution, 150 $\mu \mathrm{L}$ of acetonitrile and an additional $75 \mu \mathrm{L}$ of $0.1 \%$ formic acid aqueous solution were added, respectively. The solution was vortexed in 30 seconds followed by standing in 5 minutes under cooling temperature. After this, each sample was centrifuged at $3000 \mathrm{rpm}$ under $4^{\circ} \mathrm{C}$ in 10 minutes. After the centrifugation, 120 $\mu \mathrm{L}$ aliquot of supernatant for each sample solution in the $1.5 \mathrm{~mL}$ test tube was 
transferred into other $1.5 \mathrm{~mL}$ test tube and additional $80 \mu \mathrm{L}$ of $0.1 \%$ formic acid aqueous solution was added followed by being vortexed in 30 seconds, respectively. The final solution was 10 -flod dilution from its original blood plasma sample. $1 \mu \mathrm{L}$ of each final solution was injected into the LC/MS system by an autosampler.

As shown in Figure 1, tryptophan is degraded in many products.

4) Comparison of TRP metabolites in plasma and serum

Measurements of plasma and serum levels of TRP metabolites

We recruited young healthy men for the determinations of TRP in plasma and serum. Young healthy doctors were recruited at Hamamatsu University School

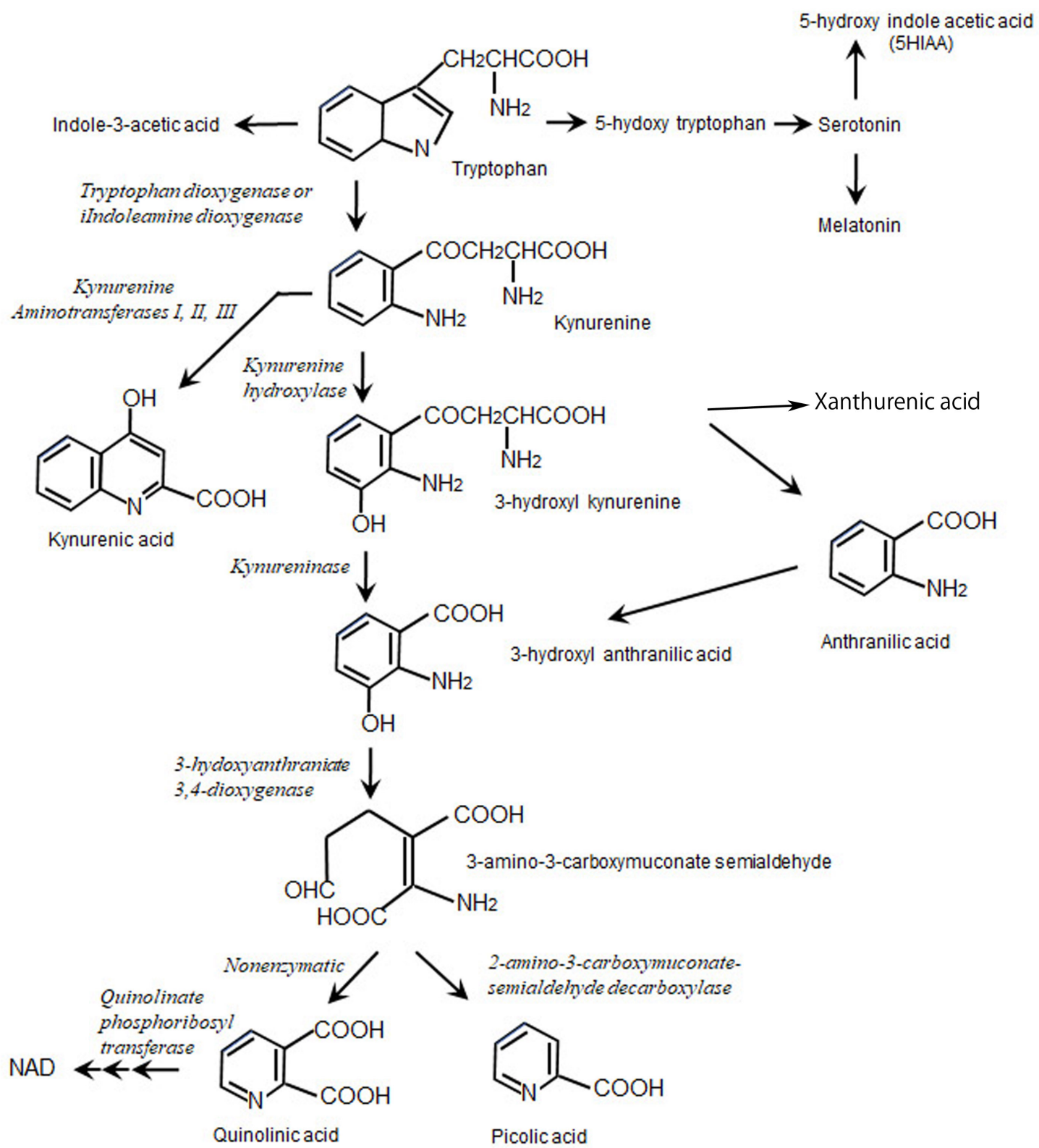

Figure 1. Shows tryptophan metabolic pathways. 
of Medicine Hospital.

The ethical committee of the University approved the experiments.

Table 2 shows the background of participants for the preparation of plasma and serum.

\section{Results}

1) Measurements of plasma levels of TRP metabolites in healthy young and old men and women

Figure 2 shows plasma levels of TRP. TRP levels of old women were significantly lower than those of young men and women and old men.

Figure 3 indicates that there were no significant differences in 5-HT levels among young and old men and women.

Standard deviations of data were so large, no statistical significance was shown among 4 groups.

Figure 4 shows plasma levels of 5-HIAA.

Plasma levels of 5-HIAA were higher in young women and old men than in young men and old women. No differences were shown in plasma levels of 5 -HIAA between young men and old women or young women and old men.

Figure 5 shows plasma levels of indole-3-acetic acid.

Plasma levels of IAA were higher in young women and old men than in young men and old women. No differences were shown in plasma levels of IAA between young men and old women or young women and old men.

Figure 6 shows that plasma levels of kynurenine were higher in young women and old men than in young men and old women.

Table 2. Background of healthy male participants $(n=10)$.

\begin{tabular}{ccc}
\hline age & height $(\mathrm{m})$ & weight $(\mathrm{kg})$ \\
\hline $34.9(27-42)$ & $1.70(1.68-1.79)$ & $66(62-84)$
\end{tabular}

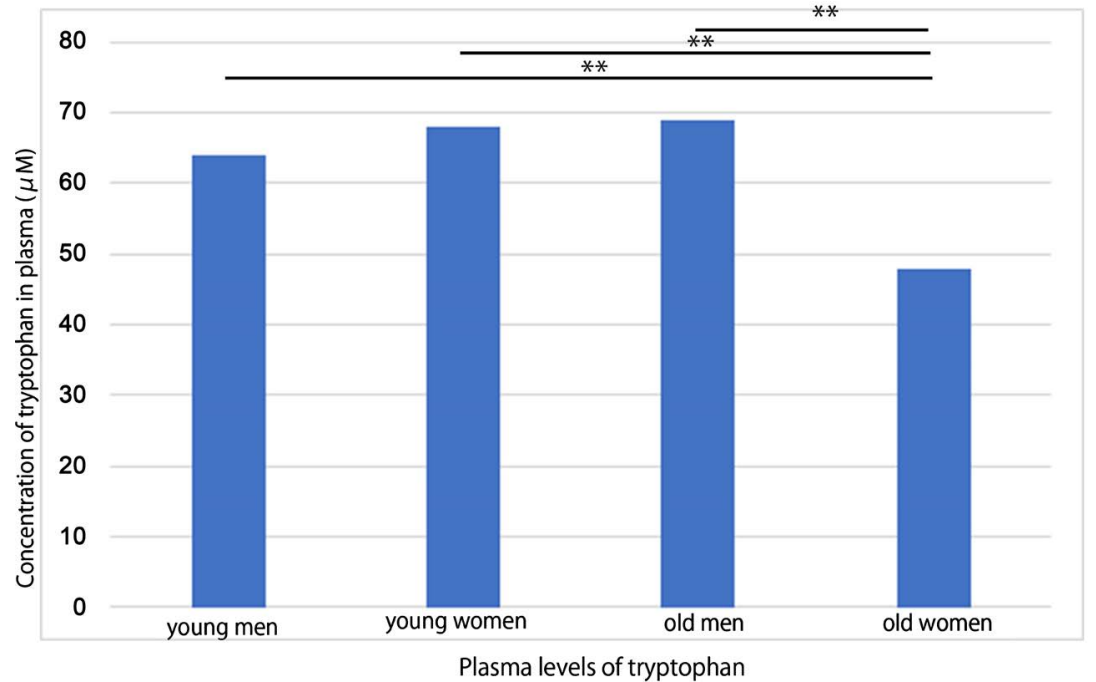

Figure 2. Plasma levels of TRP. ${ }^{* *} \mathrm{p}<0.01$. 


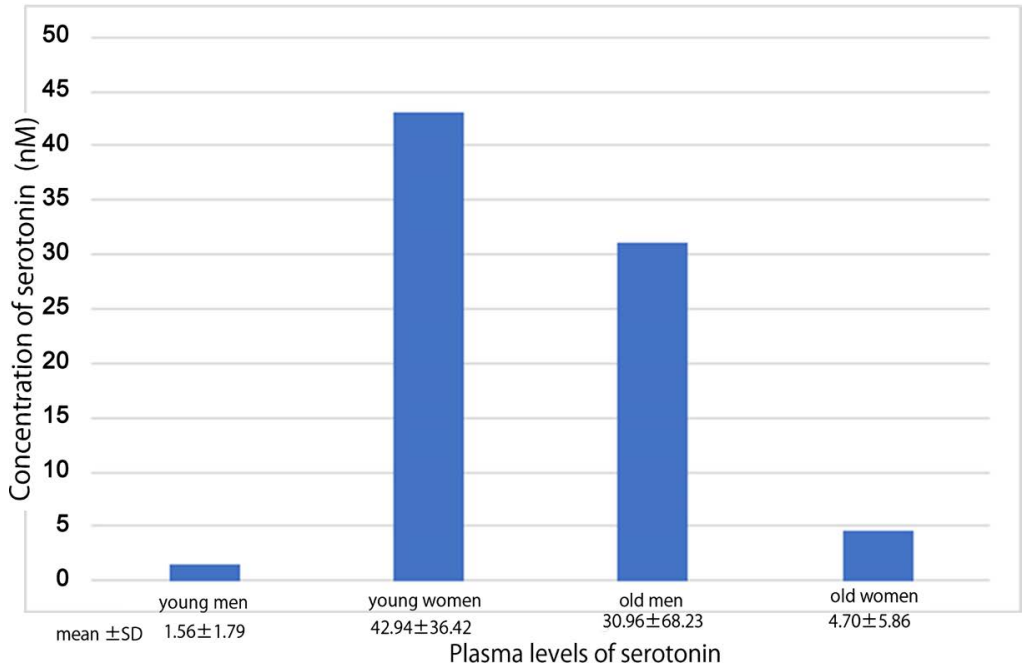

Figure 3. Plasma levels of 5-HT.

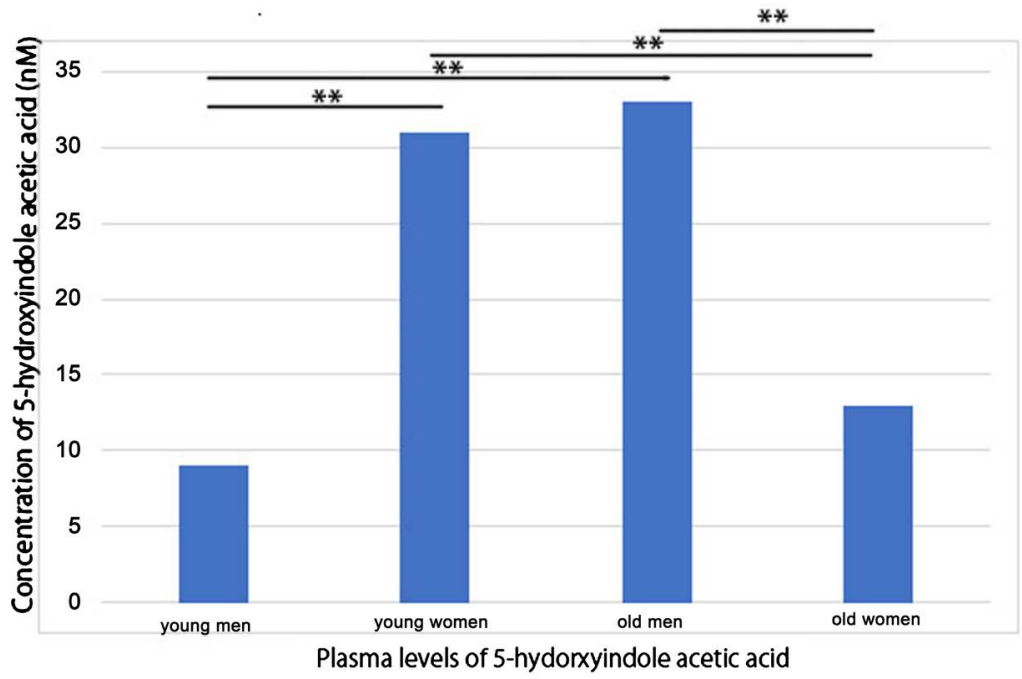

Figure 4. Plasma levels of 5-HIAA.

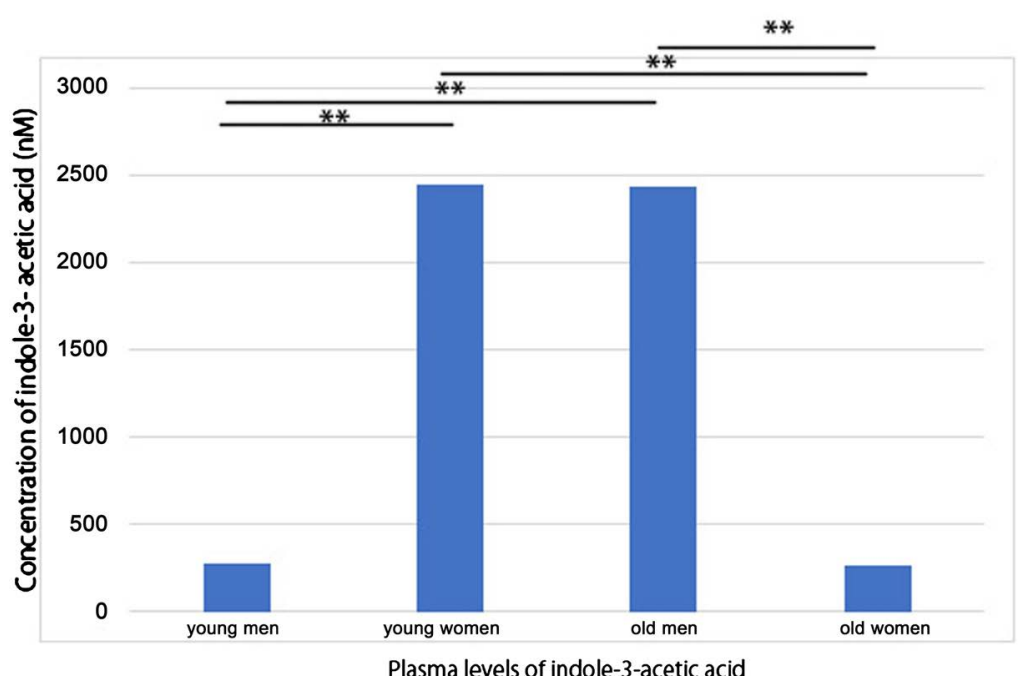

Figure 5. Plasma levels of indole-3-acetic acid. 


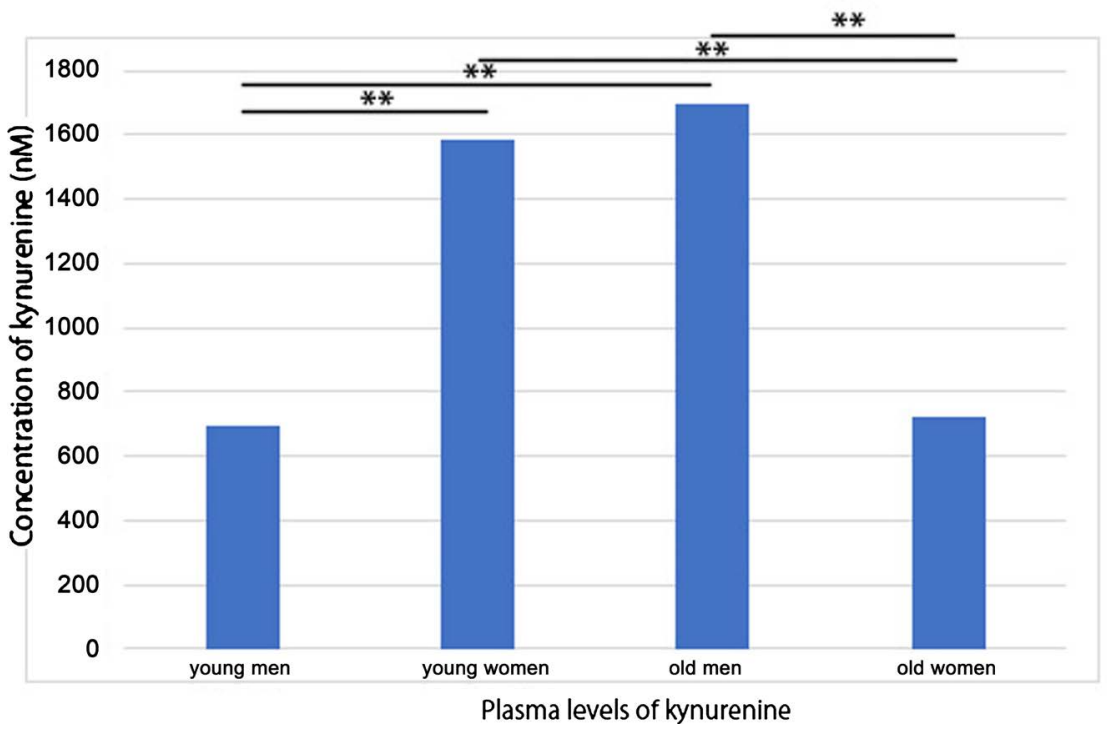

Figure 6. Plasma levels of kynurenine.

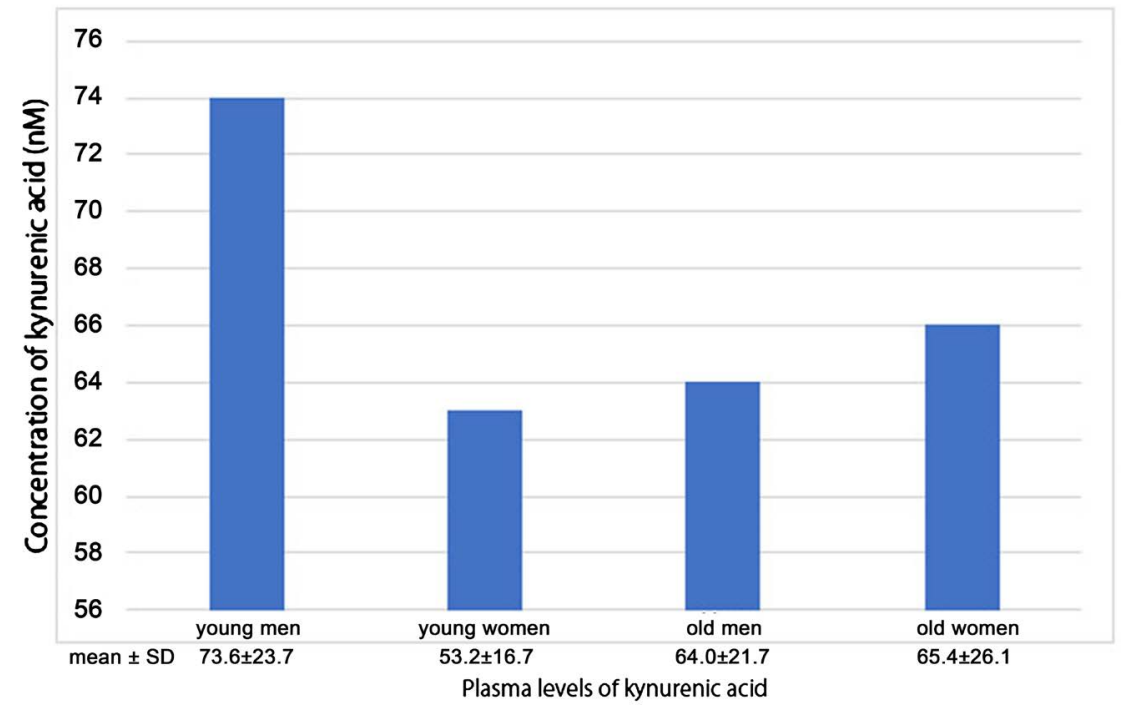

Figure 7. Plasma levels of kynurenic acid.

Figure 7 shows that there were no statistical differences in plasma levels of KNA among young and old men and women.

Figure 8 shows that plasma levels of anthranilic acid were significantly higher in old men than young men and women and old women.

Figure 9 shows that plasma levels of 3-HKN were higher in young men and old women than young women and old men.

Figure 10 shows that plasma levels of xanthurenic acid were higher in young men and old women than young women and old men.

2) Comparison of TRP metabolites levels in plasma and serum.

Figure 11 shows that TRP levels were lower in plasma than in serum. Most of other metabolites were higher in serum than in plasma except $3 \mathrm{HKY}$ and QA. Since serotonin is stored in platelets, serotonin levels were far higher in serum than plasma. 


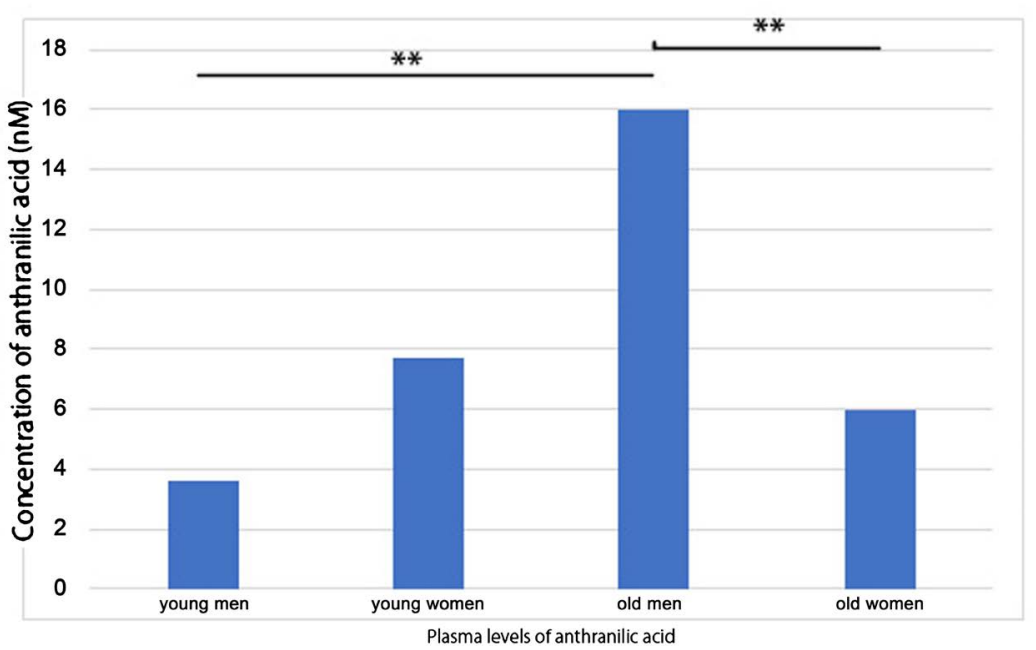

Figure 8. Plasma levels of anthranilic acid.

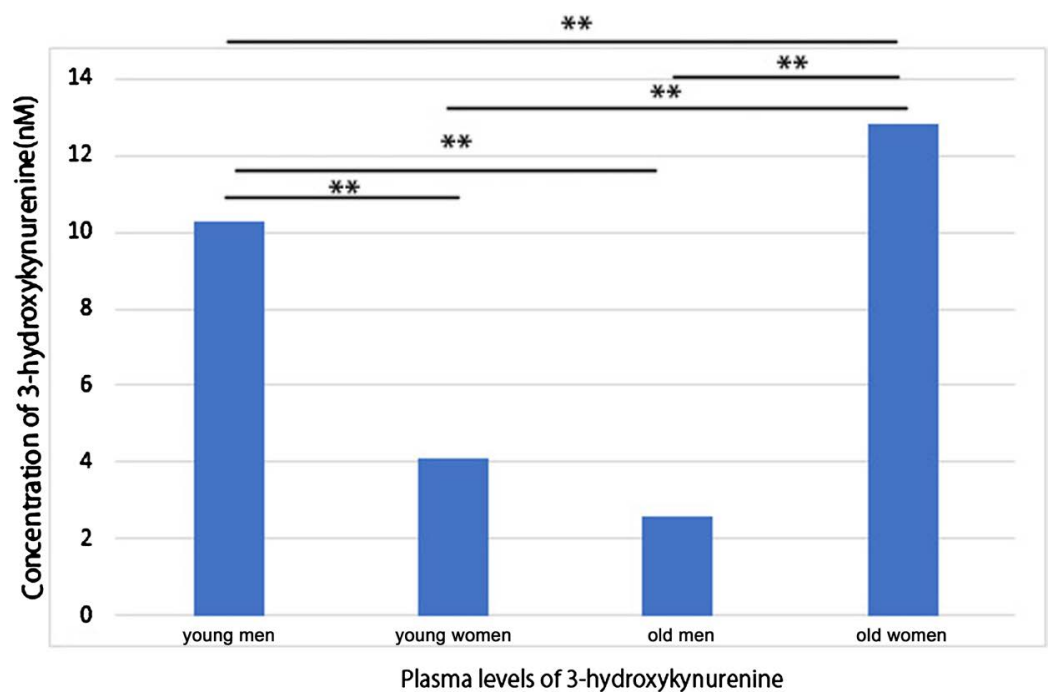

Figure 9. Plasma levels of 3-hydroxykynurenine.

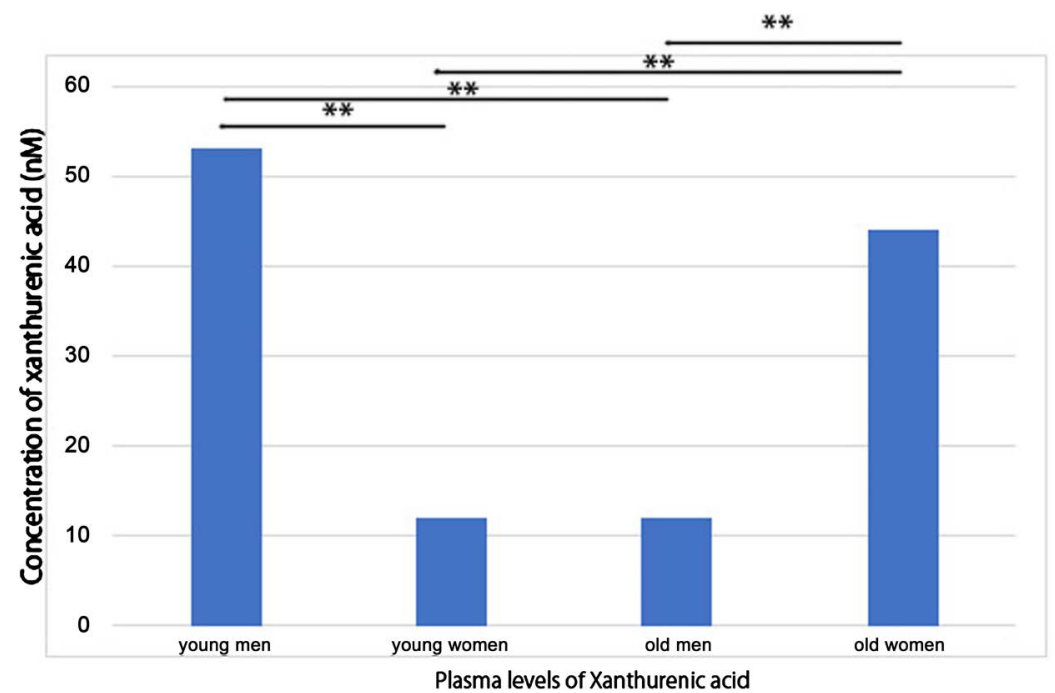

Figure 10. Plasma levels of xanthurenic acid. 


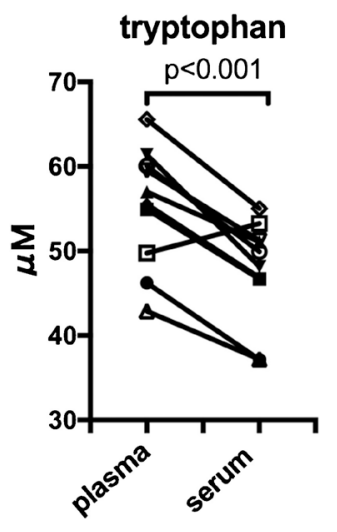

indole-3-acetic acid
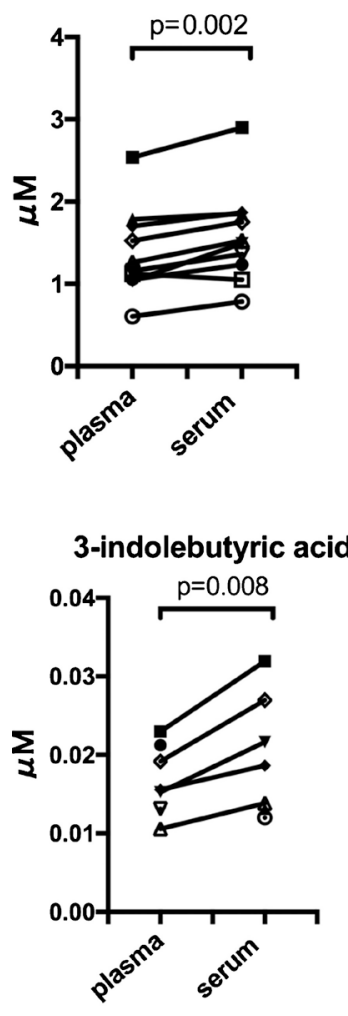

quinolinic acid

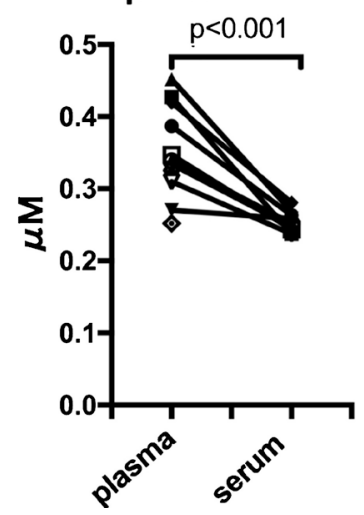

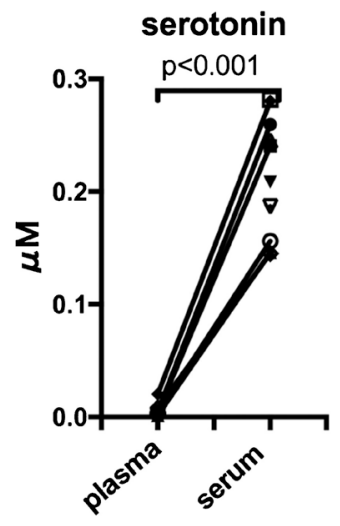
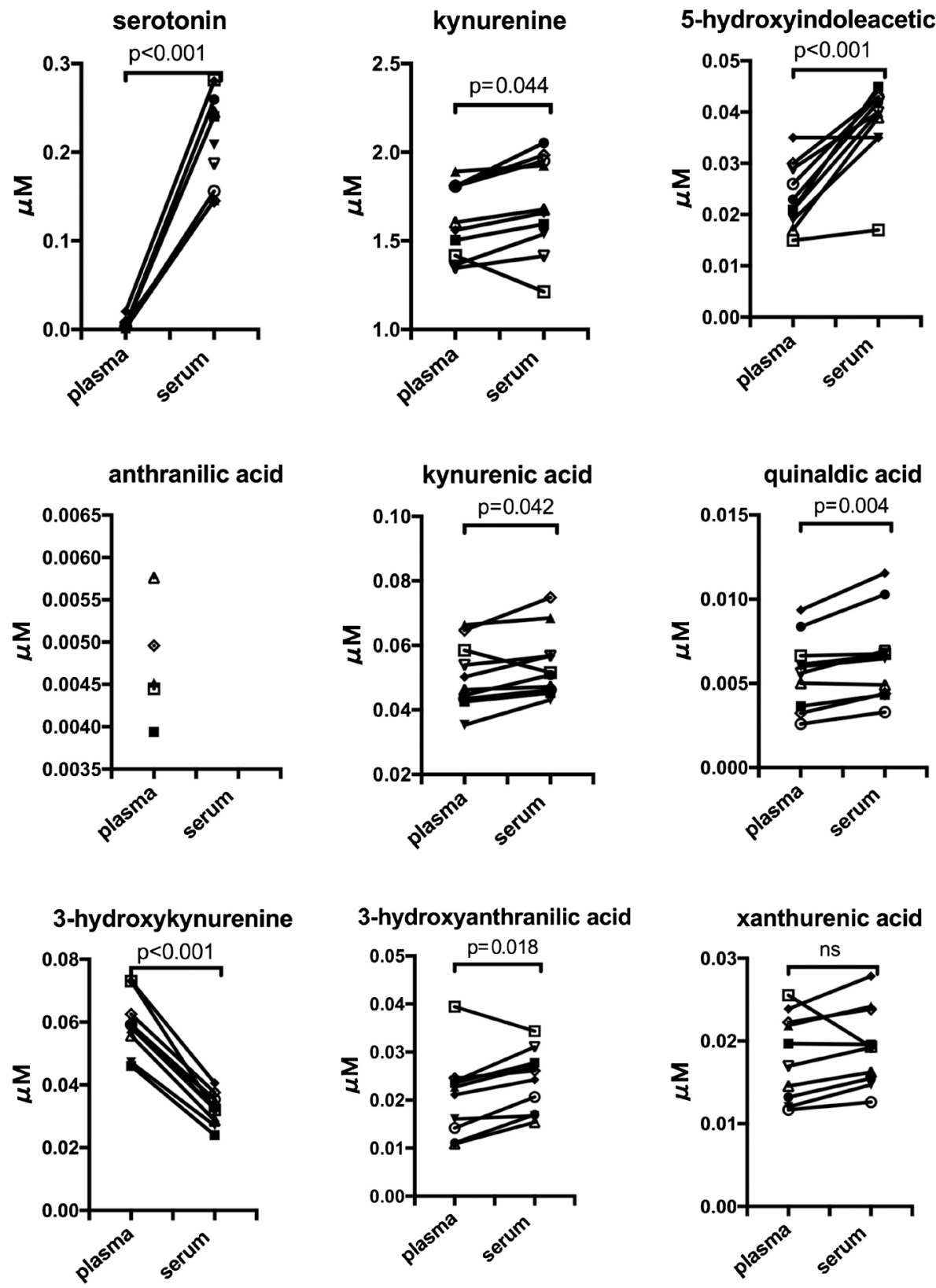

Figure 11. Levels of various TRP metabolites in plasma and serum. 


\section{Discussion}

Scientists have paid attention to tryptophan (TRP) due to its role in the production of 5-HT in the brain, which is an important transmitter regulating mood, anxiety, cognition or memory [1]. Serotonin is further converted to 5-HIAA, or $\mathrm{N}$-acetylserotonin or melatonin, which controls circadian rhythmicity. However, as stated in the INTRODUCTION, the majority of free TRP are degraded through the kynurenine pathway. The final product is nicotinamide adenine dinucleotide (NAD+), which is now under investigation for a therapeutic target of several diseases [15] Kynurenine (KYN) and its metabolites play various roles in the central nervous system. They stimulate or suppress the functions of glia cells. Defects in KYN signaling are shown in a mouse model of Alzheimer disease or Huntington disease. In nutrition, there are some papers indicating that insulin regulates the metabolism of carbohydrate, lipid, protein, and amino acid [16]. Proteolysis and associated release of amino acids are inhibited by insulin and insulin stimulate amino acid uptake and protein synthesis in skeletal muscle [16] [17]. High insulin levels were shown to stimulate skeletal muscle protein synthesis [18]. As to individual amino acids, we have shown that plasma levels of isoleucine, leucine, aspartic acid, glutamic acid are higher in T2DM patients and arginine, asparagine, cystine, and glutamine are lower in T2DM patients compared with those in old men [12]. It has been shown that plasma levels of phenylalanine, valine, leucine, isoleucine, and tyrosine increased and those of histidine and glutamine decreased in hyperglycemia [19].

As stated in the INTRODUCTION, serotonin (5-HT) has been known to be important for etiology and treatments of psychiatric diseases. Although KYN pathway is also involved in many nervous functions [20], we do not discuss the matter in details here.

As to relationships between serotonin levels and depression, we analyzed plasma levels of TRP metabolites in patients of depression [11].

Although the concentration of 5-HT has been considered to be low in depressive patients [21], 5-HT concentration in the brains of suicide victims was not low [22]. Therefore, it is not known if 5-HT concentration is decreased in the brains of depressive patients.

We decided to measure TRP metabolites in patients of monopolar depression. We could not measure 5-HT in plasma in the majority of patients.

Although plasma serotonin levels and 5-HT /TRP ratio were low in depressive patients, the levels of 5-HIAA/TRP were not lower in depressive patients. This result indicates that 5 -HT is degraded to 5 -HIAA in depressive patients almost to the same extent to healthy old and young women.

We measured the levels of KYN in healthy old men and young women, and depressive patients. TRP seems to be degraded to KNY pathways to the same extent in normal and depressive patients.

These results suggest that in depressive patients 5-HT was quickly degraded to 5-HIAA, and this seems to be a reason of low 5-HT levels in depressive patients. 
As to a relationship between serotonin pathway and KNY pathway, Lapin IP suggested that in depression tryptophan 2,3-dioxygenase in the liver shunted metabolism of serotonin away from 5-HT production to KNY production, resulting in serotonin deficiency [23]. KNY, QN and 3-HKNY were shown to be anxiogenic and KNYA were angiolytic [24]. From these results, he tried to explain the effects of anti-depressive drugs.

Our results do not support this hypothesis. Metabolites of KNY pathways were not high in depressive patients.

There have not been enough studies as to 5-HT levels in the brain of patients of bipolar depression. Serotonin levels in cerebrospinal fluids of patients of bipolar depression were shown to be high [25] or normal [26]. So it seems to be very important to discriminate monopolar and bipolar depression to study roles of serotonin in the pathogenesis of disease.

Our results show that plasma 5-HT levels were low and metabolites of KNY pathway were not changed in patients of monopolar depression.

In the field of immunotherapy of cancer, roles of $\mathrm{KYN}$ pathway have been studied extensively. Some tumors express high levels of the PD-1 (programmed cell death-1)-biding ligand (PD-L1), and initial trials of anti-PD-1 therapy found that PD-L1 expression correlated well with response to therapy [27].

In order for checkpoint therapy more effective, IDOI (indoleamine dioxygenase inhibitor) has been used. The rationale for the use of IDOI is that kynurenine is immunosuppressive, thus preventing effective T-cell attacks to tumors.

Although immune checkpoint blockade is effective, this therapy is not generally curative, and only a fraction of patients respond. So a multicombination immunotherapy including IDOI and PD-L1 blockade is introduced for inducing synergistic antitumor immunity [28] [29] [30].

Figure 12 shows that IDOI inhibit the suppression of immune response of

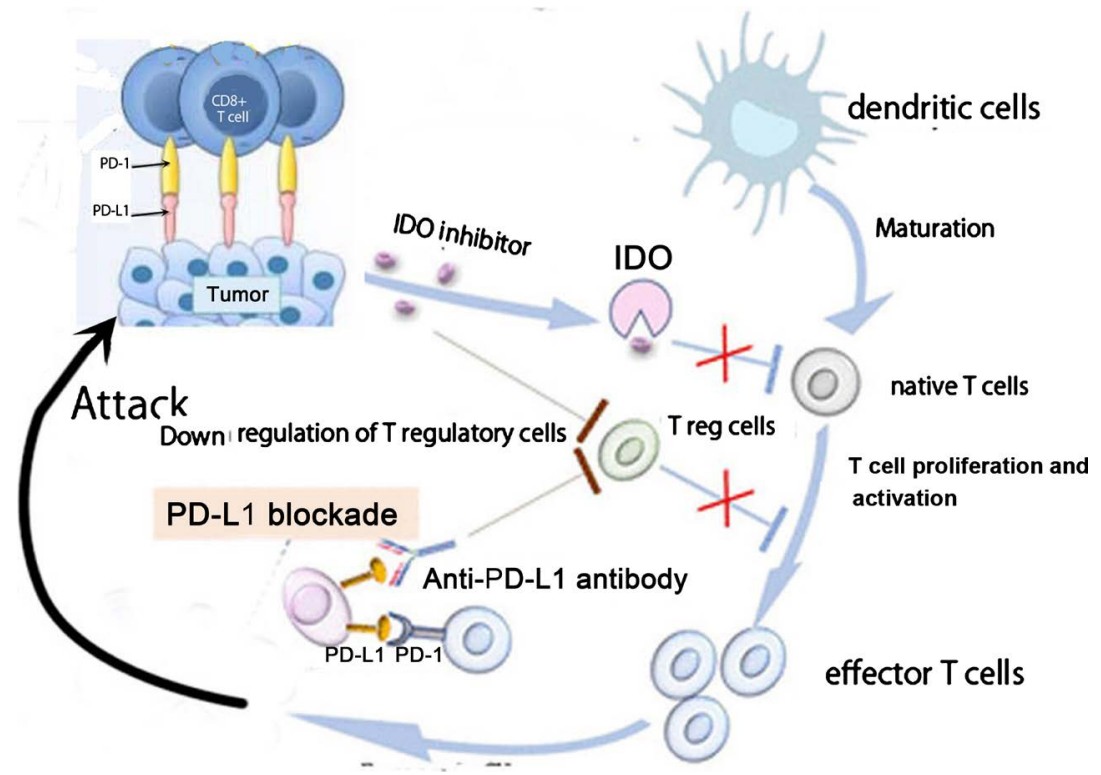

Figure 12. Schematic pictures of effects of using IDOI and PD-L1 blockade (modified from [31]). 
Native T-cells. IDOI also block the downregulation of $\mathrm{T}$ regulatory cells. Use of anti-PD-1 antibody enhances cytotoxic effects of T-cells

For such therapy, it is very important to examine the effects of age, gender, and clot formation.

The present studies may help researchers and clinicians to better analyze the efficacy of IDOI therapies.

\section{Statistics}

The results are presented as means \pm SD. Statistical significance of the differences between groups was calculated according to one-way ANOVA. When ANOVA indicated a significant difference $(\mathrm{P}<0.05)$, the mean values were compared using Tukey's least significant difference test at $\mathrm{P}<0.05$.

\section{Acknowledgements}

Experiments were designed and performed by all of the authors. AT wrote a manuscript. Statistical analyses were done by FS. All authors read the manuscript and approved the final version. All the authors had responsibilities for the final content. No conflicts of interest for any author.

\section{Financial Support}

This study was supported by grants by NPO "International Projects on Food and Health".

\section{Conflicts of Interest}

The authors declare no conflicts of interest regarding the publication of this paper.

\section{References}

[1] Cervenka, I., Agudelo, L.Z. and Ruas, J.L. (2017) Kynurenines: Tryptophan's Metabolites in Exercise, Inflammation, and Mental Health. Science, 357, eaaf9794. https://doi.org/10.1126/science.aaf9794

[2] Yates, C.A. and Herbert, J. (1976) Differential Circadian Rhythms in Pineal and Hypothalamic 5-HT Induced by Artificial Photoperiods or Melatonin. Nature, 262, 219-220. https://doi.org/10.1038/262219a0

[3] Stone, T.W., Stoy, N. and Darlington, L.G. (2013) An Expanding Range of Targets for Kynurenine Metabolites of Tryptophan. Trends in Pharmacological Sciences, 4, 136-143. https://doi.org/10.1016/j.tips.2012.09.006

[4] Malyszko, J., Urano, T., Yan, D., Serizawa, K., Kozima, Y., et al. (1994) Foot Shock-Induced Changes in Blood and Brain Serotonin and Related Substances in Rats. The Japanese Journal of Physiology, 44, 35-47. https://doi.org/10.2170/jiphysiol.44.35

[5] Malyszko, J., Urano, T., Takada, Y. and Takada, A. (1995) Amino Acids, Serotonin, and 5-Hydroxyindoleacetic Acid Following Foot Shock in Rats. Brain Research Bulletin, 36, 137-140. https://doi.org/10.1016/0361-9230(94)00178-4

[6] Pawlak, D., Takada, Y., Urano, T. and Takada, A. (2000) Serotonergic and Kynu- 
renic Pathways in Rats Exposed to Foot Shock. Brain Research Bulletin, 53, 197-205. https://doi.org/10.1016/S0361-9230(00)00252-5

[7] Okuno, E., Nishikawa, T. and Nakamura, M. (1996) Kynurenine Aminotransferases in the Rat. Localization and Characterization. Advances in Experimental Medicine and Biology, 398, 455-464. https://doi.org/10.1007/978-1-4613-0381-7_71

[8] Nakagami, Y., Saito, H. and Katsuki, H. (1996) 3-Hydroxykynurenine Toxicity on the Rat Striatum in Vivo. The Japanese Journal of Pharmacology, 2, 183-186. https://doi.org/10.1254/jjp.71.183

[9] Price, L.H., Charney, D.S., Delgado, P.L., Goodman, W.K., Krystal, J.H., et al. (1990) Clinical Studies of 5-HT Function Using I.V. L-Tryptophan. Progress in Neuro-Psychopharmacology \& Biological Psychiatry, 14, 459-472. https://doi.org/10.1016/0278-5846(90)90002-X

[10] Stone, T.W. (1993) Neuropharmacology of Quinolinic and Kynurenic Acids. Pharmacological Reviews, 45, 309-379.

[11] Takada, A., Shimizu, F. and Masuda, J. (2018) Measurement of Plasma Tryptophan Metabolites: Clinical and Experimental Application for Depression and Stress States Assessment. In: Drăgoi, C.M. and Nicolae, A.C., Eds., Melatonin-Molecular Biology, Clinical and Pharmaceutical Approaches, IntechOpen, London, 143-160. https://doi.org/10.5772/intechopen.78560

[12] Matsuoka, K., Kato, K., Takao, T., Ogawa, M., Ishii, Y., Shimizu, F., Masuda, J. and Takada, A. (2017) Concentrations of Various Tryptophan Metabolites Increase in Patients of Diabetes Mellitus Compared to Healthy Aged Male Adults. Diabetology International, 8, 69-75. https://doi.org/10.1007/s13340-016-0282-y

[13] Shimizu, F., Ishii, Y., Ogawa, M., Takao, T., Matsuoka, K., Kato, K. and Takada, A. (2018) Plasma Levels of Tryptophan Metabolites in Healthy Young and Old Men and Women, and Patients of Type 2 Diabetes Mellitus (T2DM). Obesity: Open Access, 4. https://doi.org/10.16966/2380-5528.138

[14] Takada, A., Shimizu, F. and Masuda, J. (2019) Plasma Levels of Tryptophan Metabolites in Patients of Type 2 Diabetes Mellitus. In: Watson, R. and Preedy, V., Eds., Bioactive Food as Dietary Interventions for Diabetes, 2nd Edition, Academic Press, Cambridge, 265-276. https://doi.org/10.1016/B978-0-12-813822-9.00017-5

[15] Cantó, C., Menzies, K.J. and Auwerx, J. (2015) NAD+ Metabolism and the Control of Energy Homeostasis: A Balancing Act between Mitochondria and the Nucleus. Cell Metabolism, 22, 31-53. https://doi.org/10.1016/j.cmet.2015.05.023

[16] Felig, P. (1975) Amino Acid Metabolism in Man. Annual Review of Biochemistry, 44, 933-955. https://doi.org/10.1146/annurev.bi.44.070175.004441

[17] Jefferson, L.S., Li, J.B. and Rannels, S.R. (1977) Regulation by Insulin of Amino Acid Release and Protein Turnover in the Perfused Rat Hemicorpus. The Journal of Biological Chemistry, 252, 1476-1483.

[18] Biolo, G., Williams, B.D., Fleming, R.Y. and Wolfe, R.R. (1999) Insulin Action on Muscle Protein Kinetics and Amino Acid Transport during Recovery after Resistance Exercise. Diabetes, 48, 949-957. https://doi.org/10.2337/diabetes.48.5.949

[19] Robinson, M.M., Soop, M., Sohn, T.S., Morse, D.M., Schimke, J.M., et al. (2014) High Insulin Combined with Essential Amino Acids Stimulates Skeletal Muscle Mitochondrial Protein Synthesis While Decreasing Insulin Sensitivity in Healthy Humans. The Journal of Clinical Endocrinology \& Metabolism, 99, E2574-E2583. https://doi.org/10.1210/jc.2014-2736

[20] Chen, Y. and Guillemin, G.J. (2009) Kynurenine Pathway Metabolites in Humans: Disease and Healthy States. International Journal of Tryptophan Research, 2, 1-19. 
https://doi.org/10.4137/IJTR.S2097

[21] Mann, J.J., Arango, V., Marzuk, P.M., Theccanat, S. and Reis, D.J. (1989) Evidence for the 5-HT Hypothesis of Suicide. A Review of Postmortem Studies. The British Journal of Psychiatry, 155, 7-14. https://doi.org/10.1192/S000712500029171X

[22] Oquendo, M.A., Sullivan, G.M., Sudol, K., Baca-Garcia, E., Stanley, B.H., et al. (2014) Toward a Biosignature for Suicide. American Journal of Psychiatry, 171, 1259-1277. https://doi.org/10.1176/appi.ajp.2014.14020194

[23] Lapin, I.P. (1973) Kynurenines as Probable Participants of Depression. Pharmakopsychiatrie, Neuro-Psychopharmakologie, 6, 273-279.

https://doi.org/10.1055/s-0028-1094391

[24] Lapin, I.P. (1998) Antagonism of Kynurenic Acid to Anxiogens in Mice. Life Sciences, 63, 231-236. https://doi.org/10.1016/S0024-3205(98)00404-4

[25] Pålsson, E., Sellgren, C., Rydén, E., Kizza, R., Pelanis, A., Zetterberg, H., Blennow, K. and Landén, M. (2017) Cerebrospinal Fluid Monoamine Metabolite Profiles in Bipolar Disorder, ADHD, and Controls. Journal of Neural Transmission (Vienna), 124, 1135-1143. https://doi.org/10.1007/s00702-017-1746-3

[26] Berrettini, W.H., Nurnberger, J.I., Scheinin, M., Seppala, T., Linnoila, M., Narrow, W., Simmons-Alling, S. and Gershon, E.S. (1985) Cerebrospinal Fluid and Plasma Monoamines and Their Metabolites in Euthymic Bipolar Patients. Biological Psychiatry, 20, 257-269. https://doi.org/10.1016/0006-3223(85)90055-1

[27] Keenan, T.E., Burke, K.P. and Van Allen, E.M. (2019) Genomic Correlates of Response to Immune Checkpoint Blockade. Nature Medicine, 25, 389-402. https://doi.org/10.1038/s41591-019-0382-x

[28] Soliman, H.H., Minton, S.E., Han, H.S., et al. (2016) A Phase I Study of Indoximod in Patients with Advanced Malignancies. Oncotarget, 7, 22928-22938. https://doi.org/10.18632/oncotarget.8216

[29] Richards, T. and Brin, E. (2018) Cell Based Functional Assays for IDO1 Inhibitor Screening and Characterization. Oncotarget, 9, 30814-30820. https://doi.org/10.18632/oncotarget.25720

[30] Cohen, E.E.W., Soulières, D., Le Tourneau, C., et al. (2019) Pembrolizumab versus Methotrexate, Docetaxel, or Cetuximab for Recurrent or Metastatic Head-and-Neck Squamous Cell Carcinoma (KEYNOTE-040): A Randomised, Open-Label, Phase 3 Study. The Lancet, 393, 156-167. https://doi.org/10.1016/S0140-6736(18)31999-8

[31] Yan, M., Liu, Y., Zhu, X., Wang, X., Liu, L., Sun, H., Wang, C., Kong, D. and Ma, G. (2019) Nanoscale Reduced Graphene Oxide-Mediated Photothermal Therapy Together with IDO Inhibition and PD-L1 Blockade Synergistically Promote Antitumor Immunity. ACS Applied Materials \& Interfaces, 11, 1876-1885.

https://doi.org/10.1021/acsami.8b18751

\section{Abbreviation}

tryptophan (TRP), serotonin (5-HT), 5-hydroxyindole acetic acid (5-HIAA), indole-3-acetic acid (IAA), kynurenine (KYN), xanthurenic acid (XA), anthranilic acid (AA), kynurenic acid (KNA), 3-hydroxykynurenine (3-HKN), indoleamine dioxygenase (IDO), tryptophan dioxygenase (TDO). 